\title{
Knowledge regarding post-exposure prophylaxis amongst nurses in an African context
}

\author{
Lufuno Makhado ${ }^{1}$, Boipelo Seekane ${ }^{2}$ \\ ${ }^{1}$ School of Health Sciences, University of Venda, Thohoyandou, South Africa \\ ${ }^{2}$ Ganyesa Hospital, Ganyesa, North West Province, South Africa
}

\begin{abstract}
Occupational exposure to human immunodeficiency virus (HIV) among nurses had been reported to be a major challenge in South African and broader African context. There seems to be an increase in the level of exposure and uptake of post-exposure prophylaxis (PEP) in Africa. This had warranted the need to establish a systematic review on the level of knowledge among nurses regarding PEP in the African continent. The search engines used included: EBSco Host (North-West University [NWU] library), Sabinet, Google Scholar, ScienceDirect, and PubMed. The search was limited to the current decade (2008-2018), including articles that are in English. Keywords used were: 'knowledge, 'post-exposure prophylaxis/PEP', 'HIV', and 'nurs*'. Thematic evaluation to pick out commonalities of the applications covered in this review have been achieved. The themes identified from the review were: poor knowledge regarding PEP, receiving training on PEP for HIV, immediate steps to take after exposure to infected blood, exposure to HIV, and not taking PEP.

There is a strong need to increase the awareness and knowledge regarding PEP as well as to implement training programs to train nurses on the process of PEP in healthcare settings, to increase knowledge on PEP, and thereby decrease seroconversion of HIV. y.
\end{abstract}

HIV AIDS Rev 2020; 19, 1: 8-15 DOI:

Key words: Africa, HIV, knowledge, awareness, nurses, post-exposure prophylaxis.

\section{Introduction}

Occupational exposure to human immunodeficiency virus (HIV) among nurses had been reported to be a major challenge in South African as well as other African countries [1]. 'Nurses' in this systematic review shall mean all category of nurses, thus student nurse, enrolled nursing auxiliary, enrolled nurse as well as professional nurse. Nurses face a great challenge due to occupational HIV exposure, as they provide baseline care to diverse, unique individuals, thus nurses are treating people who are HIV-positive, HIV-nega- tive, and with unknown HIV status. Occupational exposure contributes to the burden of HIV in the workplace, with increased morbidity and mortality on healthcare workers who provide care to people living with HIV [2]. As of December 31, 2013, 58 validated occupational transmissions of HIV and one hundred and fifty viable transmissions had been reported in the United States. Of these, solely one validated case has been suggested since 1999. Underreporting of instances had been described by the National Institute for Occupational Safety and Health (NIOSH) as a matter of concern, and this is so given that case reporting is voluntary [3].

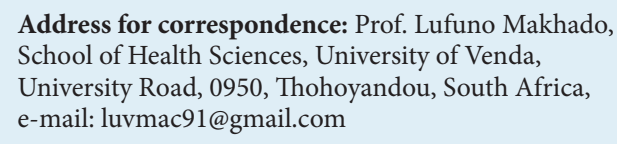

Article history:

Received: 09.04.2019

Received in revised form: 17.05.2019

Accepted: 20.05.2019

Available online: 30.01 .2020
International Journal of HIV-Related Problems

HIV \& AIDS

R e v i e w 
The World Health Organization (WHO) outlined that each year there are three million cases of percutaneous exposure in healthcare settings [4-6]. Percutaneous, contact, and mucous membranes were revealed to be the customary types of exposure. It was further highlighted that percutaneous exposure via a needlestick is the most common mode of occupational HIV transmission [1, 2]. Transmission via mucous membranes as well as through abraded skin is the least common mode of occupational HIV transmission. The average risk of percutaneous exposure following HIV exposure is estimated to be $0.3 \%$ and $0.09 \%$ after exposure to mucous membranes [8]. The studies showed that occupational exposures occur predominantly in developing countries, although they are infrequently reported $[9,10]$.

Occupational exposure is explained as the contact with an infected patient's blood or bodily fluids by healthcare personnel at work [17]. When there is an occupational exposure to HIV, post-exposure prophylaxis (PEP) is a routine method to be implemented as it prevents $81 \%$ seroconversion $[4,6,11,12]$. PEP comprises of first aid, counselling, risk assessment, HIV testing, established informed consent of the exposed individual as well as by maintaining confidentiality of the findings, with continuous counselling and support to promote adherence $[9,13,14]$. PEP does not guarantee that one cannot get infected by HIV after exposure, as it is not $100 \%$ effective, but it can decrease the risk of HIV transmission by $81 \%[13,15,16]$.

PEP was defined as a precautionary medication of 2-3 antiretroviral (ARV) drugs administered following exposure to a pathogen, to prevent or reduce the likelihood of HIV transmission $[1,16,18]$. PEP should be administered/initiated as early as possible, but at least within one hour of exposure, with the latest being within 72 hours after exposure [19]. The ARVs, however, should be taken for a period of 28 days after exposure [20,21]. PEP is still the best prophylaxis and currently the only method used to prevent seroconversion after exposure. There seems to be an increase in the level of exposure and uptake of PEP in Africa. This had warranted the need to establish a systematic review on the level of knowledge among nurses regarding PEP in the African continent.

\section{Material and methods}

The study conducted was documented in a study protocol. The types of studies that were required for this review were cross-sectional descriptive studies, which were used to discover the knowledge of nurses regarding PEP. Participants were nurses in Africa. Interventions were finding out information regarding nurse's knowledge on PEP. The outcome measures were for nurses to have sufficient knowledge on PEP.

The search engines that were used were: EBSco Host (NWU library), Sabinet, Google Scholar, ScienceDirect, and PubMed. The search was limited to the current decade (2008-2018) and the articles included were English written. Keywords that were used were: 'knowledge', 'post-exposure
prophylaxis/PEP', 'HIV', and 'nurs*'. PRISMA (preferred reporting items for the systematic review and meta-analysis) tool was used for the review process and results. The search was conducted twice with the help of the NWU-based librarian through databases/search engines, and this was followed by a manual search of reference list of eligible studies. The titles, abstracts, methods, and results of different studies were screened by the investigators independently for inclusion of potential articles as well as to remove duplicates. The PRISMA flow diagram was used as a guidance tool for the systematic review [22].

\section{Study selection}

The study selection was accomplished by using the PRISMA flow diagram of 2009 to identify data included and excluded, and their rationale for exclusion. Initially, all studies were screened using their titles and abstracts. Furthermore, the studies, which could not be excluded from this review had their full texts evaluated. In order to achieve this, all selected items had their full texts sought, with their eligibility in terms of inclusion criteria double-checked. The evaluation of titles, abstracts, and full texts as well as manual searches from selected articles' reference list was performed between September and October 2018, with the last search on the 18 October 2018 with the help of a librarian. All studies selected did not need approval from the authors for use as they were available publicly. Only the full text of the selected studies that met the inclusion criteria were subjected to quality appraisal (Figure 1).

\section{Appraisal of selected studies}

Appraisal of results was conducted using the Critical Appraisal Skills Programme, a cohort study checklist to assess the articles thoroughly and systematically, in order to ensure their relevance and trustworthiness [23]. Seven studies were assessed to confirm their relevance; the results are conveyed in Table 1.

\section{Results}

The total search results screened were 18,111, with 79 duplicates. Results that were relevant to the topic by title and abstract were 111. Screening of titles and abstracts were conducted independently by the researchers after removing duplicates. Full-text articles that were considered to meet the inclusion criteria were 23 in total. Articles that were included for the review were seven. Some articles were relevant, but not included as some of them were: not published in English, older than 10 years (not relevant to the 2008-2018 era), and not relevant to the keywords that were used in the study. Articles that were considered for review for the evaluation of the articles needed to describe/fulfill the following terms: knowledge and post-exposure prophylaxis/PEP. Table 2 provides the characteristics of the selected studies. 


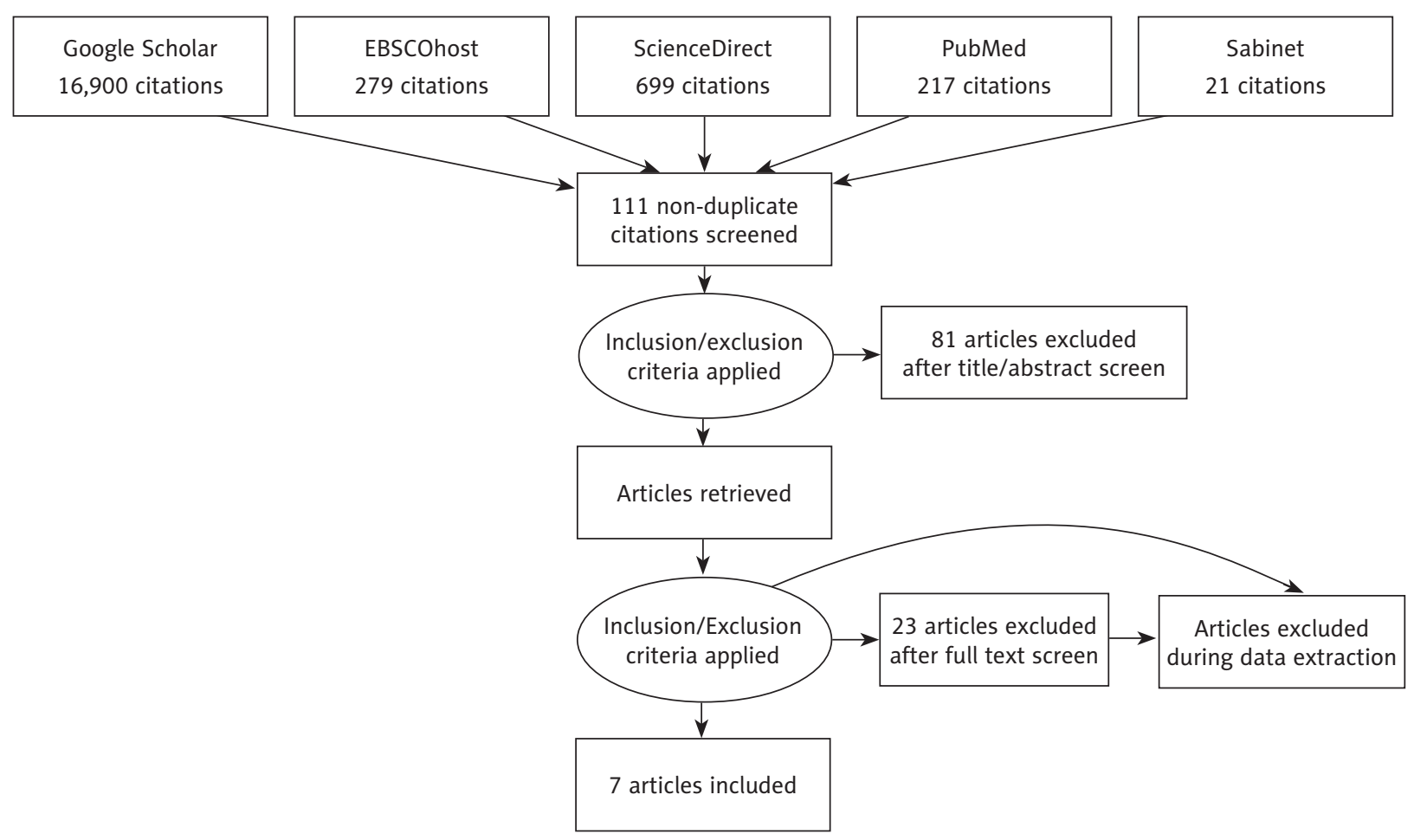

Figure 1. PRISMA flow diagram

Table 1. Appraisal of studies using CASP

\begin{tabular}{l|c|c}
\hline Authors and year & Study design & Assessment of studies \\
\hline Aminde et al., 2015 & Cross-sectional study & $6 / 10(60 \%)$ \\
\hline Dhital et al., 2017 & A descriptive design & $5 / 10(50 \%)$ \\
\hline Lamichanne et al., 2012 & Descriptive cross-sectional research design & $5 / 10(50 \%)$ \\
\hline Mabina et al., 2018 & Cross-sectional descriptive study & $7 / 10(70 \%)$ \\
\hline Makhado et al., 2016 & Cross-sectional descriptive design & $8 / 10(80 \%)$ \\
\hline Olowabi et al., 2012 & Cross-sectional study design & $6 / 10(60 \%)$ \\
\hline Sendo et al., 2014 & Cross-sectional descriptive study & $8 / 10(80 \%)$ \\
\hline
\end{tabular}

Thematic evaluation to pick out commonalities of the applications covered in this review have been achieved. The themes identified from the review were:

- level of knowledge regarding $\operatorname{PEP}(n=7)$ [2, 4, 6, 8, 24-26],

- receiving training on PEP for $\operatorname{HIV}(n=4)[4,6,8,25]$,

- immediate steps to take after exposure to infected blood $(n=2)[6,8]$

- exposure to HIV and not taking PEP $(n=5)$ [2, 4, 24-26].

\section{Level of knowledge regarding post-exposure prophylaxis}

Poor information concerning PEP suggest that there will be excessive possibilities of poor adherence as well as seroconversion of HIV. If there is little understanding regarding PEP, there will also be an increase in mortality and morbidity rates, particularly in developing countries. Poor knowledge regarding PEP means that nurses may have little or no information regarding PEP and as a result, nurses may not take PEP (due to lack of knowledge) and this may place them at risk of transmitting HIV. In a study that was conducted in Cameroon, $73.7 \%$ of the nurses had a lack of information concerning PEP [4]. From a study conducted Bharatpur, a sample of 50 nurses were used and from the sample, out of which $40 \%$ did not know the correct meaning of PEP and most of the participants not knowing the correct regimen for PEP (46\%); 50\% of the participants did not know of the duration of PEP and $66 \%$ of participants had pre-knowledge on PEP [8]. Research conducted in Nepal concluded that nurses only sought information regarding PEP by self-learning, with $78 \%$ on a sample of 425 nurses, $48 \%$ through co-workers, and $11 \%$ based on working experience [6]. About $68 \%$ of participants had fair level of knowledge on PEP [6]. On the contrary, a study 


\begin{tabular}{|c|c|c|c|c|}
\hline 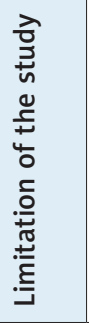 & 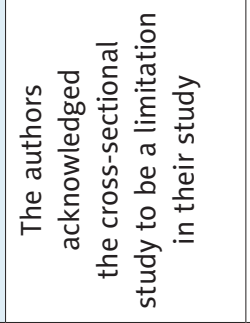 & 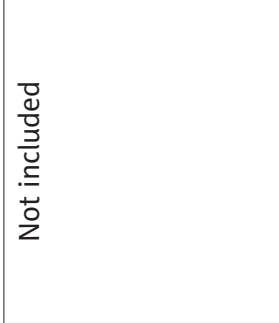 & 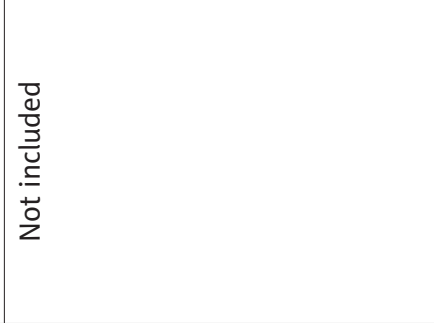 & 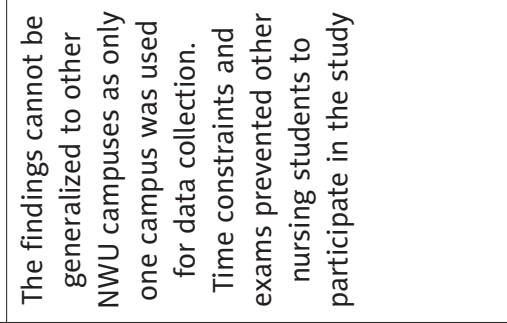 \\
\hline 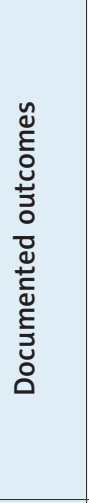 & 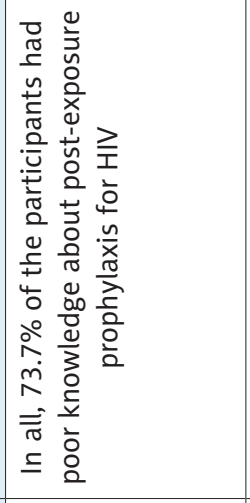 & 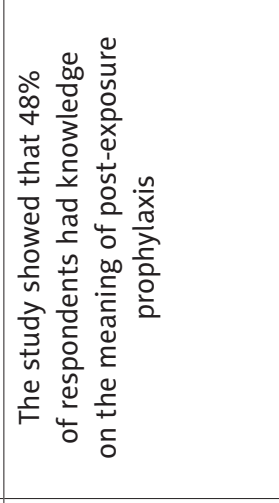 & 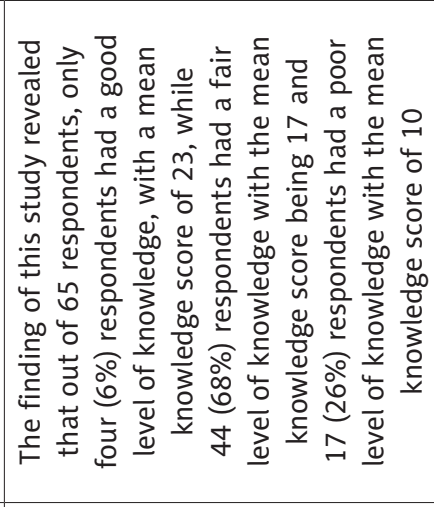 & 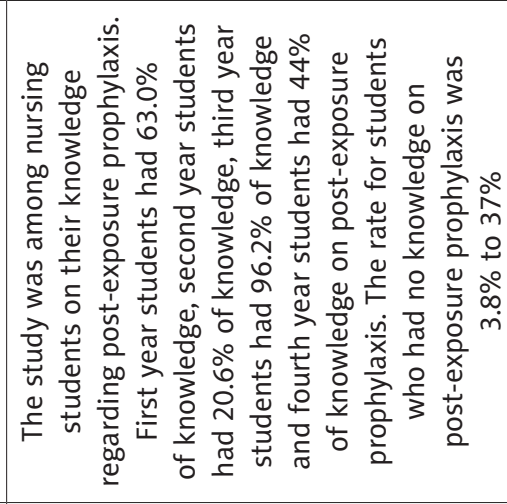 \\
\hline 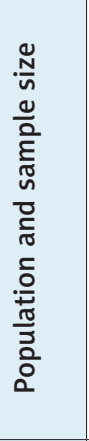 & 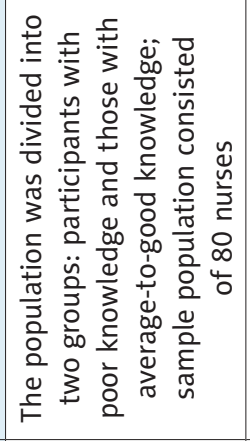 & 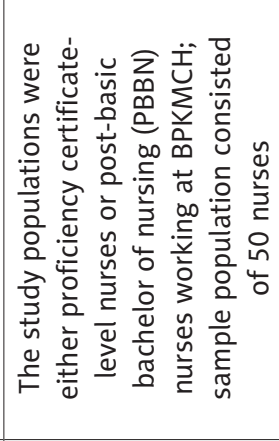 & 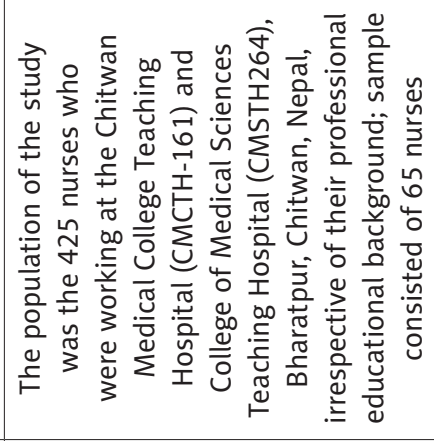 & 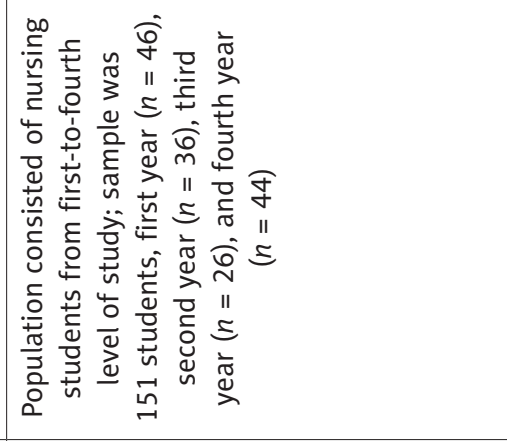 \\
\hline 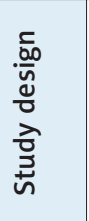 & 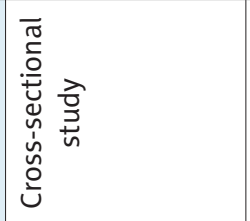 & 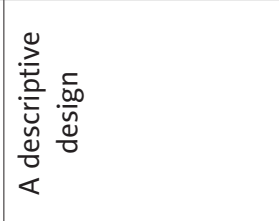 & 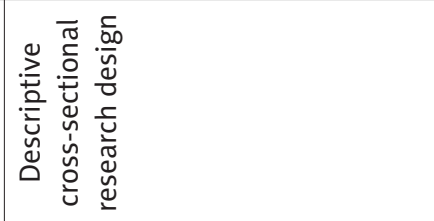 & 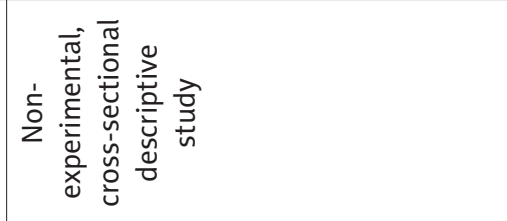 \\
\hline $\begin{array}{l}\frac{2}{3} \\
\frac{1}{0} \\
0\end{array}$ & 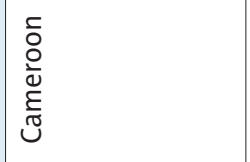 & 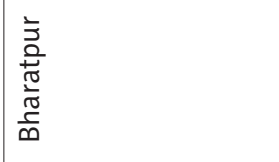 & $\begin{array}{l}\overline{\frac{\pi}{0}} \\
\frac{\mathrm{d}}{2}\end{array}$ & $\begin{array}{l}\text { 吾 } \\
\text { 总 } \\
\text { 心 }\end{array}$ \\
\hline 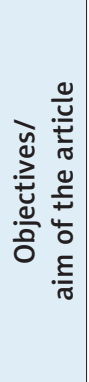 & 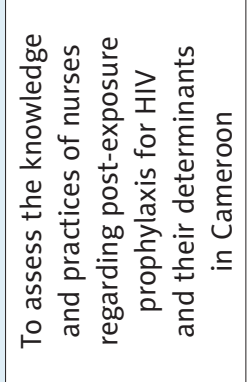 & 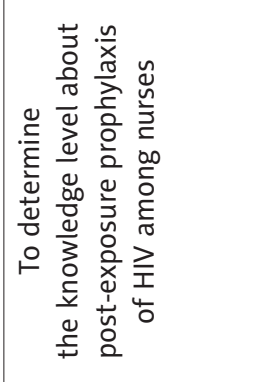 & 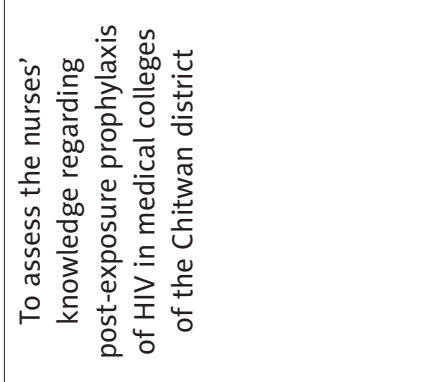 & 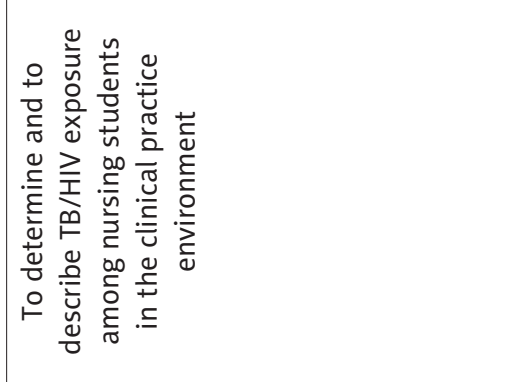 \\
\hline 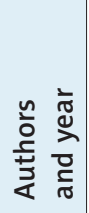 & 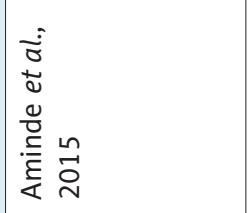 & 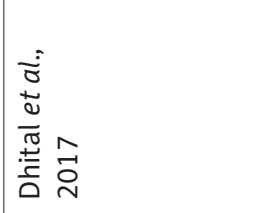 & 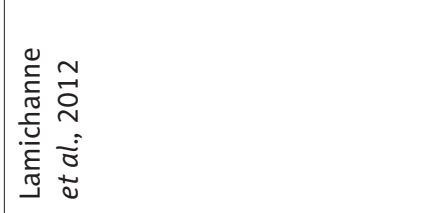 & 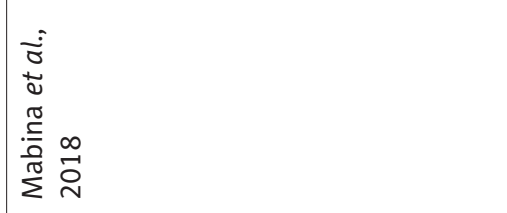 \\
\hline
\end{tabular}




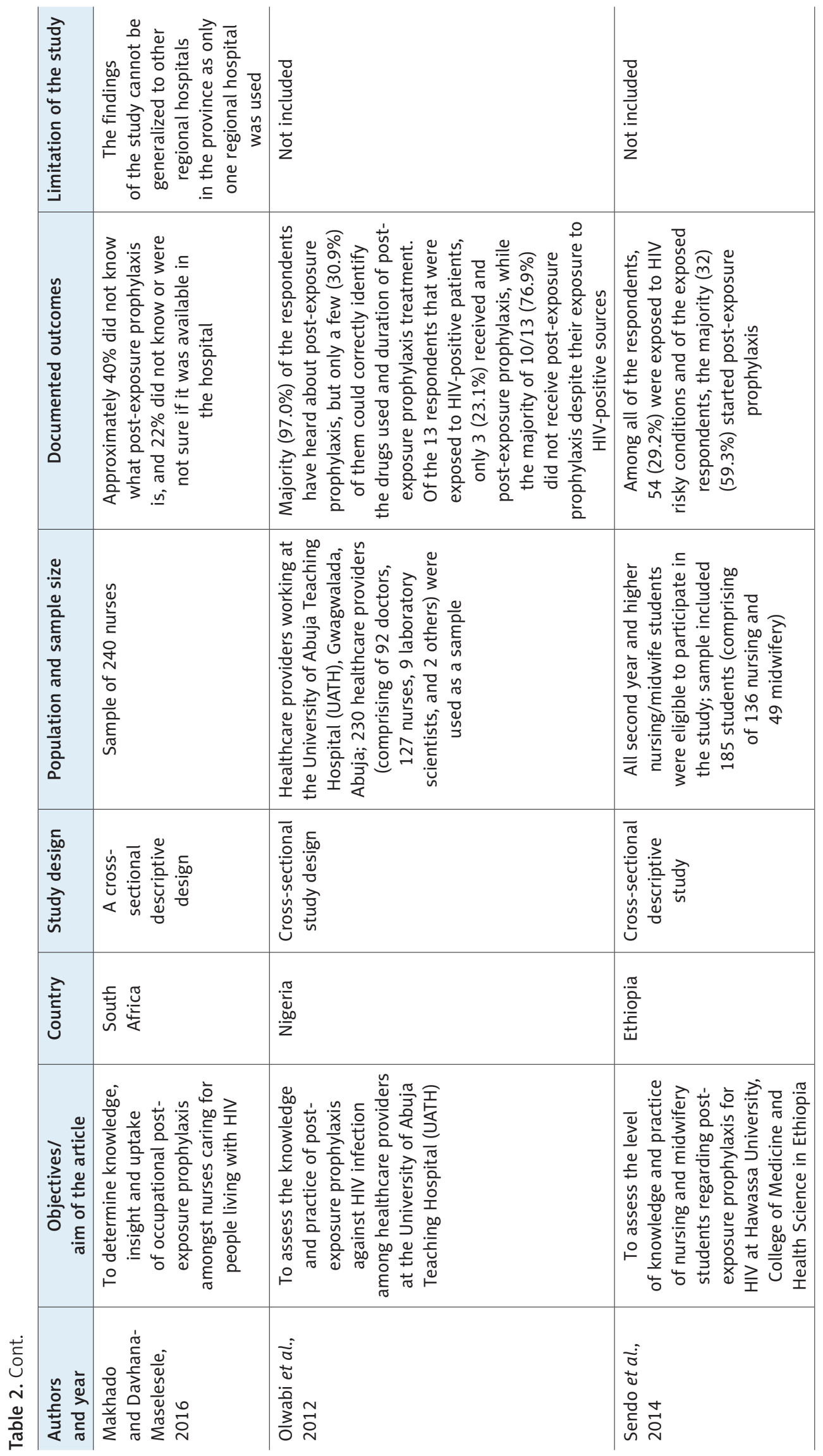


in the south African context also highlighted that $40 \%$ of nurses did not know what PEP is, which proves that only $60 \%$ of the participants were familiar with PEP [2]. A Nigerian study reported that $95.3 \%$ of nurses were acquainted with PEP [25], while another Ethiopian study indicated that $49.2 \%$ of student nurses and $17.8 \%$ of midwives were aware of PEP, with approximately $63 \%$ of inadequate knowledge on PEP [26]. A study among nursing students on their knowledge regarding PEP revealed that first year students had $63.0 \%$ of knowledge, second year students had $20.6 \%$ of knowledge, third year students had $96.2 \%$ of knowledge, and fourth year students had $44 \%$ of knowledge on PEP [24]. The number of students who had no knowledge on PEP ranged between $3.8 \%$ and $37 \%$, respectively. This indicates that knowledge of nurses regarding PEP varied between different cadre of nurses and context.

\section{Receiving training on post-exposure prophylaxis}

Training nurses on PEP may assist them to enhance and transfer their knowledge to others. This approach might motivate the majority of nurses to consider opting for PEP after HIV exposure in a healthcare setting, for example via a needle stick injury. Receiving training on PEP includes: first aid, assessment of risk, counselling, HIV testing on informed consent, confidentiality, and providing psychological support. Thorough training on PEP may enhance adherence as well as disease prevention. Although the number of PEP training differed across study sites, it was evident from two studies that $12.5 \%$ and $44.8 \%$ nurses had received training on PEP, which leaves $87.5 \%$. and $56.2 \%$ remaining untrained, respectively $[4,25]$. This was also highlighted and emphasized through two studies, which indicated that nurses did not receive any training on PEP $[6,8]$.

\section{Immediate steps to take after exposure to infected blood}

Following basic steps after exposure is crucial to reduce the rapid transmission of the HIV pathogen in the bloodstream. This can be achieved by rinsing the exposed part of the body with running water. This approach does not reduce the risk of HIV transmission, but it is a basic step to take after exposure to an infected patients' blood or bodily fluids, i.e. amniotic fluid. Some nurses may not have adequate knowledge on how to take immediate steps after exposure to infected blood. This was evident from two studies that $39.9 \%$ and $48 \%$ reported that the exposed site is washed with soap and water, respectively $[6,8]$, and $94.5 \%$ reported that the exposed areas should be rinsed with water or normal saline [8].

\section{Exposure to HIV and not taking post- exposure prophylaxis}

When one is exposed to HIV and does not sought PEP as a preventative measure, the risk of transmission will be great. Additionally, it will also increase the rate of morbidity and mortality in the continent. Majority of nurses are exposed to HIV, but are failing to take PEP due to various reasons, which include inadequate knowledge regarding PEP, and some are reluctant to take PEP due to the perceived side effects. About $18 \%$ of the respondents who were exposed to HIV did not receive PEP because their source of HIV transmission was negative [4]. There were about $37 \%$ of participants who did not receive PEP when they needed it, and $42 \%$ participants who did not seek PEP because they did not want it, $16 \%$ did not have enough information about PEP, $12 \%$ did not know where to go, and $20 \%$ were afraid of the process [2]. Nurses and co-workers usually share their experiences; those who are inexperienced, for instance, (in this case) those who never took PEP, may be afraid of the side effects of PEP or the outcome of the treatment. This statement is supported by reviewed studies where about $20 \%$ of the participants were afraid of the outcome of PEP [2], $45.5 \%$ of respondents were concerned about the side effects of PEP [26], while $6.5 \%$ to $36.8 \%$ of respondents were afraid to go through the process [4]. Some nurses are unaware of the hospital policy that highlights the necessity to take PEP after occupational exposure, thus $15.9 \%$ and $86.4 \%$ of participants were unaware of the hospital policy for PEP, respectively $[4,26]$. Some nurses assume that the source is HIV-non-reactive and they decide to not take PEP, as $17 \%$ of participants who were exposed to HIV did not seek PEP due to assuming that the source is HIV-negative and $10 \%$ believed that they can never be infected with HIV [25].

\section{Discussion}

Nurses offer a baseline care to patients; as a result, they are the healthcare workers who are at the greatest risk of occupational exposure, especially through needle pricks. There is a way to reduce the likelihood of HIV transmission after exposure, but most nurses have little knowledge on this aspect, while some have knowledge but opt to not seek PEP as they are afraid to go through the treatment process.

The studies included in this paper revealed that nurses had inadequate knowledge regarding PEP [2, 4, 6, 8, 24-26]. Despite varied level of knowledge nurses have regarding PEP, PEP remains the only ARV treatment regimen intended to reduce the occupational transmission of HIV [7]. It is vital to educate nurses on PEP for successful prevention of HIV in the workplace [5]. Many respondents were aware of PEP, but still have inadequate information regarding PEP; for instance, many nurses can define PEP, but may not know the initiation period, the duration of taking the regimen as well as the type of antiretroviral drugs used $[1,19]$.

Although precautionary measures to prevent exposure to infected blood via safe practices are in place, occupational exposure still occurs and are mostly underreported [27]. An increase in the numbers of exposed healthcare workers attending health facilities as patients was evidenced by HIV sero-prevalence rates and healthcare workers have justified concerns about the risk of accidental exposure [10]. Deter- 
mining the knowledge and practice of PEP among healthcare workers would identify their needs and the next line of action when exposed to HIV [15].

Four studies have conveyed that nurses have limited knowledge on PEP, with a few who are aware of PEP $[6,8,24$, 26]. Although the percentage of people who have knowledge about PEP were above average in some studies, it is worrying as, regardless of percentage, it is a high number of affected nurses in the midst of inadequate workforce. This exemplification of nurses having little knowledge regarding PEP may lead to high seroconversion of HIV and therefore, promote an increase in mortality and morbidity rates among nurses in the African context. Nurses need to be educated on PEP to prevent transmission of HIV in the workplace as well as to be able to seek PEP in due course and for $100 \%$ adherence.

Four other studies indicated that nurses had training on PEP at a rate of $0 \%$ to $48 \%[4,6,8,25]$. A conclusion may be drawn from these statistics that nurses who have received training on PEP are in minority. As a result, nurses will not be able to implement the accurate procedure when managing an individual exposed to HIV and this may lead to poor adherence as well as defaulting from the PEP treatment, while in turn, there will be high seroconversion among nurses $[1,2]$. Such procedures that require training on PEP comprise of counselling, HIV screening, first aid, risk assessment, HIV testing, established informed consent of the exposed individual as well as maintaining confidentiality of the findings and to offer continuous counselling and support to promote adherence $[9,13,15,18$, 27]. Nurses need to be trained on PEP, so that they can acquire relevant knowledge and skills on the management thereof.

According to seven reviewed studies, most nurses knew the immediate steps to be taken after exposure to HIV, which included rinsing the exposed site with soap and water, squeezing the exposed site for blood in case of needle prick injury as well as to rinse the eye with water or normal saline in case of blood splash or body fluids in the eye [2, 4, 6, 8, 24-26]. These precautionary measures after exposure to HIV are very beneficial to the healthcare workers as they may reduce the likelihood of transmission of the pathogen in the bloodstream, although they may not prevent the transmission of HIV. Furthermore, though many nurses know the immediate steps to take after exposure to infected blood or body fluids, more information on universal precautionary measures should be stressed to all those who have pre-knowledge and students or newly qualified nurses [1, 12, 20, 27].

It is evident that few nurses seek PEP, but most do not take PEP because they assume that the source is negative; they believe they will not get infected, they are afraid to go through the process, they are not aware of the hospital policy regarding PEP, and some just reported that they do not need PEP after exposure [2, 4, 24-26]. Respondents had varying reasons with regard to not seeking PEP, but the origin of the problem lies with fact that they do not have the adequate and relevant knowledge regarding PEP. Failure to take PEP within 72 hours after exposure places one at a higher risk of seroconversion or at danger of contracting HIV. The importance of taking PEP after exposure should be enforced to all nurses and should be compulsory, if the healthcare system needs to keep and promote a healthy workforce $[9,18]$. Furthermore, extra monitoring and follow-ups should be maintained to promote adherence throughout the course of PEP [4]. This can be achieved by in-service training, posters on health facilities, and regular reviewing of PEP policy at health facilities.

\section{Limitations}

The review was only limited to studies done in Africa and cannot be generalized to other contexts outside Africa. It should also be noted that some of the reasons provided for not taking PEP were not followed up by the reviewed studies, which limited further explanation in this review.

\section{Conclusions}

Knowledge regarding PEP is essential amongst nurses, as it provides a baseline of treatment to individuals who are exposed to HIV. Many nurses are aware of PEP, but do not exactly have the relevant knowledge regarding PEP. Additionally, training is very limited amongst nurses and in some countries, nurses do not receive training for PEP at all. The psychological factor of nurses needs also to be taken into account, as they are afraid to go through the process of PEP and are afraid of the results. Provision of counselling as well as offering the support to those that are exposed should be taken into consideration to enhance $100 \%$ adherence. There is a need to increase awareness and knowledge regarding PEP as well as implementing training programs to train nurses on the process of PEP in healthcare settings to increase knowledge on PEP, and thereby decreasing the level of seroconversion of HIV.

\section{Recommendations}

- Guidelines and policies should be reviewed and implemented for the management of PEP to provide standards and direction for the knowledge and use of PEP.

- PEP services should be available 24/7 and there should be access to health facilities at any time of the day, for example, access to PEP at night or over weekends as PEP should be initiated within 72 hours after exposure and it is always best to start PEP within one hour after HIV exposure.

- There should be compulsory use of protective equipment in healthcare facilities and there should be an appointed person or team who monitors the use of protective equipment or clothing among nurses and healthcare workers to reduce the incidences of occupational exposure.

- Nurses should be educated and trained on PEP in order to possess the necessary knowledge and skills aimed at the management when one is exposed to HIV.

\section{Acknowledgement}

The authors are grateful to the North-West University library and the librarian for the assistance in the literature search within different databases. 


\section{Conflict of interest}

The authors declare no conflict of interest with respect to the research, authorship, and/or publication of this article.

\section{References}

1. Ajibola S, Akinbami A, Elikwu C, Odesanya M, Uche E. Knowledge, attitude and practices of HIV post exposure prophylaxis amongst health workers in Lagos University Teaching Hospital. Pan Afr Med J 2014; 19: 172.

2. Makhado L, Davhana-Maselesele M. Knowledge and uptake of occupational post-exposure prophylaxis amongst nurses caring for people living with HIV. Curationis 2016; 39: 1593.

3. CDC. Arkansas, Louisiana, and West Virginia Have Highest Rates of Work-related Deaths in South eastern United States. NIOSH Research Rounds 2016; 2. Available at: https://www.cdc.gov/niosh/ research-rounds/resroundsv2n4.html.

4. Aminde LN, Takah NF, Dzudie A, et al. Occupational post-exposure prophylaxis (PEP) against human immunodeficiency virus (HIV) infection in a health district in Cameroon: assessment of the knowledge and practices of nurses. PLoS One 2015; 10: e0124416.

5. Sabane HH, Dixit RR, Durge PM. Impact of knowledge about post exposure prophylaxis among nursing students - a cross sectional study. Nursing 2011; 1: 4-68.

6. Lamichanne J, Aryal B, Dhakal KS. Knowledge of nurses on post exposure prophylaxis of HIV in Medical Colleges of Chitwan district, Nepal. Int J Pharm Biol Arch 2012; 3: 1394-1399.

7. Alenyo R, Fualal J, Jombwe JJ. Knowledge, attitude and practices of staffs towards post-exposure prophylaxis for HIV infection at Mulago Hospital in Uganda. East and Central African Journal of Surgery 2009; 14: 99-102.

8. Dhital PS, Sharma S, Poudel P, Dhital PR. Knowledge regarding post exposure prophylaxis of HIV among nurses. Nursing: Research and Reviews 2017; 7: 45-50.

9. Mashoto KO, Mubyazi GM, Mushi AK. Knowledge of occupational exposure to HIV: a cross sectional study of healthcare workers in Tumbi and Dodoma hospitals, Tanzania. BMC Health Serv Res 2015; 15: 29.

10. Taegtmeyer M, Suckling RM, Nguku PM, et al. Working with risk: occupational safety issues among healthcare workers in Kenya. AIDS Care 2008; 20: 304-310.

11. McCormack S, Dunn DT, Desai M, et al. Pre-exposure prophylaxis to prevent the acquisition of HIV-1 infection (PROUD): effectiveness results from the pilot phase of a pragmatic open-label randomised trial. Lancet 2016; 387: 53-60.

12. Mill J, Nderitu E, Richter S. Post-exposure prophylaxis among Ugandan nurses: "Accidents do happen". International Journal of Africa Nursing Sciences 2014; 1: 11-17.

13. Saoud IB, Elsour IF, Elbargathi AM, Elmarak AM, Ali ES. Knowledge, attitudes and practices of health care workers in Benghazi, Libya towards post exposure prophylaxis for HIV. Ibnosina J Med BS 2013; 5: 318-323.

14. Western Cape Province Department of Health. Management \& PostExposure Prophylaxis of Potential HIV and Hepatitis B Exposure in Children, Adolescents \& Adults Guidelines. 2016

15. Adebimpe WO. Knowledge and practice of health care workers towards post exposure prophylaxis in the era of low and stable HIV prevalence in Southwestern Nigeria. Bulletin of Faculty of Pharmacy, Cairo University 2018; 56: 104-108.

16. Knapper C, Freedman A. Post-exposure prophylaxis. Medicine 2009; 37: 378-380.

17. Shaghaghian S, Pardis S, Mansoori Z. Knowledge, attitude and practice of dentists towards prophylaxis after exposure to blood and body fluids. Int J Occup Environ Med 2014; 5: 146-154.
18. Dulcie CA, Mohamed TS, Meenakshi B, Ezhil RJ. Assessment of knowledge about post exposure prophylaxis of HIV among medical, nursing and paramedical students in hospital and laboratory practice. International Journal of Basic \& Clinical Pharmacology 2017; 6: 2408-2413.

19. Katsinde TJ, Chawira M, Katsinde CS, Nyoni C. Knowledge and attitudes of university workers towards HIV and AIDS in Zimbabwe. International Journal of Law, Humanities \& Social Science 2017; 1: 1-6.

20. Lungu MM. Knowledge, utilization of HIV post exposure prophylaxis, among student nurses at Ndola School of Nursing, Ndola, Zambia. Degree of Master of Science in Nursing. University of Zambia, 2009.

21. Marjanović M, Brkić S. Knowledge of the possibilities of post-exposure prophylaxis for HIV infection. South Eastern Europe Health Sciences Journal 2013; 3: 50-57.

22. PRISMA. Transparent reporting of systematic reviews and Meta analyses; 2009. Available at: http://prisma-statement.org/PRISMAStatement/ [Accessed: 12.09.2018].

23. Critical Appraisal Skills Programme; 2018. Available at: https:// casp-uk.net/casp-tools-checklists/ [Accessed: 7.09.2018].

24. Mabina K, Morulane K, Tong G, Makhado L. TB/HIV exposure among nursing students in the clinical practice environment. Africa Journal of Nursing and Midwifery 2018; 20: 1-17.

25. Owolabi RS, Alabi P, Ajayi S, et al. Knowledge and practice of postexposure prophylaxis (PEP) against HIV infection among health care providers in a tertiary hospital in Nigeria. J Int Assoc Physicians AIDS Care (Chic) 2012; 11: 179-183.

26. Sendo EG. Assessment of level of knowledge and practice of nursing and midwifery students on HIV post exposure prophylaxis in Hawassa University, Ethiopia. J HIV Clin Sci Res 2014; 1: 1-6.

27. Odinaka KK, Edelu BO, Nwolisa CE, Amamilo IB, Amamilo IJ. Post exposure prophylaxis against human immunodeficiency virus: Awareness knowledge and practice among Nigerian Paediatricians. Nigerian Journal of Paediatrics 2016; 43: 193-196. 